\title{
Room-temperature group-IV LED based on defect-enhanced Ge quantum dots
}

\author{
Patrick Rauter, Lukas Spindlberger, Friedrich Schäffler, Thomas Fromherz, Julia Freund, and \\ Moritz Brehm* \\ Institute of Semiconductor and Solid State Physics, Johannes Kepler University Linz, Altenberger Straße 69, A-4040 \\ Linz, Austria
}

KEYWORDS: quantum dots, silicon photonics, light-emitting diode, electroluminescence, group-IV

\begin{abstract}
As recently demonstrated, defect-enhanced Ge quantum dots (Ge-DEQDs) in a crystalline Si matrix can be employed as CMOS-compatible gain material in optically pumped lasers. Due to the stability of their optical properties up to temperatures beyond $300 \mathrm{~K}$, the Ge-DEQD system is a highly promising candidate for the realization of an electrically pumped group-IV laser source for integration in a monolithic optoelectronic platform fit for room-temperature operation. We report on the realization of light-emitting diodes based on Ge-DEQDs operating at telecom wavelengths and above room temperature. The DEQD electroluminescence characteristics were studied spectrally resolved as a function of driving current and device temperature. The experimental results show that the excellent optical properties of Ge-DEQDs are maintained under electrical pumping at high current densities and at device temperatures of at least $100^{\circ} \mathrm{C}$. Furthermore, the emission intensity scales with the number of quantum dot layers embedded into the $p$-i- $n$ diode structures, thus indicating the scalability of the approach for large gain material volumes. The presented results form an essential step towards the future demonstration of a CMOS-compatible, electrically pumped room-temperature laser based on Ge-DEQDs.
\end{abstract}

Motivated by the promise of a tremendous increase in data transfer rates ${ }^{1}$ and the implementation of novel sensing functionalities on a chip ${ }^{2,3}$, the development of a monolithic, group-IV-based optoelectronic platform has recently been attracting extensive research interest. And while the development of various components for integrated Si photonics is advancing rapidly ${ }^{4-10}$, the realization of the arguably most fundamental building block for such a universal platform remains elusive: A practical, electrically pumped group-IV laser source for monolithic integration on a Si chip has yet to be demonstrated. The reason for this persistent lack of efficient silicon-based light sources is the indirect bandgap of bulk group-IV materials that requires the interaction with an additional particle to satisfy momentum conservation in an optical transition. Approaches for overcoming this fundamental issue include the implementation of Raman lasers ${ }^{11}$, the engineering of Ge layers towards a direct bandgap by applying strain ${ }^{12,13}$ or alloying with $\mathrm{Sn}^{14}$, and relaxing momentum conservation by low-dimensional carrier confinement in quantum dots ${ }^{15}$ or defect states ${ }^{16}$. As an alternative to Si-based emitters, the hybrid integration of III-V gain material on Si chips has recently been pursued with impressive results ${ }^{17-19}$. However, depending on the detailed hybrid approach several of the advantages associated with monolithic integration are lost, most fundamental among them low material cost and yield. Monolithic integration of light sources on Si photonics chips therefore remains a heavily pursued goal, and for different group-IV systems lasing under optical pumping has very recently been demonstrated ${ }^{14,15}$. For potential applications of a monolithically integrated optoelectronic platform, electrically pumped laser operation at room temperature is highly desirable. However, electrical pumping is associated with ohmic heating, calling for a gain material which is robust against high device temperatures. In the direct-bandgap $\mathrm{GeSn}^{20}$ and $\mathrm{SiGeSn}^{21}$ systems, emission quenching sets in already at cryogenic conditions for optically pumped material ${ }^{14,21}$. Strong emission quenching with increasing temperature has also been observed for electroluminescence (EL) devices based on $\mathrm{GeSn} / \mathrm{SiGeSn}$ heterostructures $^{22,23}$. 

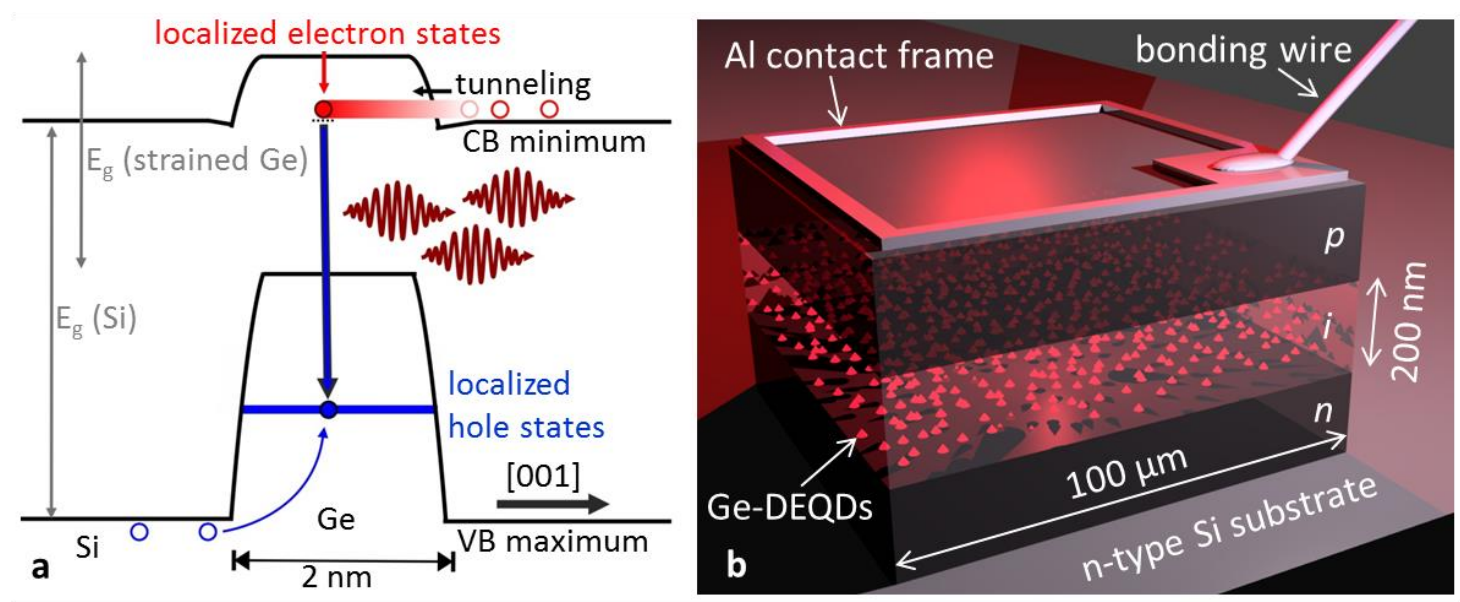

Figure 1. Ge-DEQD band scheme and LED device layout. (a) The energy profiles of the valence band maximum and conduction band minimum are shown in the [oo1] growth direction for the studied dot system. The valence band offset between the Si matrix and the Ge dot leads to a strong confinement of holes in the dot, where a localized hole level is represented by the blue line. In contrast to conventional Ge dots, in DEQDs the localization of electrons relies on the introduction of split-[10o] interstitial states deep within the Ge bandgap, which relax momentum conservation due to zerodimensional electron confinement. The latter enables efficient, spatially direct optical transitions. Both charge carrier types are provided in a forward-biased $p-i-n$ diode structure. (b) The device geometry of the DEQD LEDs is illustrated. The frameshaped top contact metallization enables emission in surface normal direction. The integration of multiple dot layers in the intrinsic device region allows scaling of the active material volume.

In stark contrast, the very recently demonstrated optically pumped laser based on defect-enhanced Ge quantum dots (Ge-DEQDs) did not show a significant drop in emission power between $10 \mathrm{~K}$ and room temperature ${ }^{15}$. This feature elevates the Ge-DEQD system to a leading candidate for the realization of a practical electrically pumped groupIV laser.

The emitter performance of DEQDs is vastly superior to that of conventional Ge quantum dots without defect engineering. Even though room-temperature EL from conventional group-IV quantum dots has been reported by several groups $^{24-29}$, optical transitions in these dots are inefficient due to a type-II band alignment ${ }^{30,31}$, and neither strong luminescence ${ }^{26,32-34}$ nor lasing has been achieved. And while conventional epitaxial group-IV dots continue to attract research interest due to the precise control over spatial dot positioning in a well-established pre-patterned growth approach ${ }^{35-37}$, their potential as a laser gain material is very limited.

In contrast to conventional Ge dots, DEQDs rely on the confinement of electrons and holes at the same location but induced by two basically different mechanisms: Holes are confined in a conventional epitaxial Ge quantum dot embedded in a silicon matrix, where the confinement potential is defined by the large valence band offset between $\mathrm{Si}$ and Ge. Electrons, on the other hand, occupy defect states deep within the Ge bandgap associated with split[10o] interstitials ${ }^{38}$ formed by the introduction of additional Ge atoms into the crystalline dot matrix ${ }^{39}$. A bandstructure scheme of the DEQD system is shown in Figure $1 \mathrm{~A}$. As evident from the calculated orbital electron densities presented in reference 39, the state introduced below the Ge conduction band edge is highly localized at the introduced defect. Therefore, due to the incorporation of the lattice defect within the Ge dot via ion bombardment, electrons and holes occupy strongly spatially overlapping states. This is in stark contrast to conventional, defect-free Ge dots, where electrons are weakly confined by strain-induced potential wells outside the Ge dot volume. To clearly point out the key feature of Ge-DEQDs: In bulk $\mathrm{Si}$ and $\mathrm{Ge}$, interband transitions are indirect in momentum space and thus weak. In conventional epitaxial Ge quantum dots, optical transitions are spatially indirect due to a type-II band alignment and again inefficient. Finally, in GeDEQDs spatially direct optical transitions are possible without additional momentum transfer, making this group-IV system capable of laser operation ${ }^{15}$. However, in the superior Ge-DEQDs system, so far only optically pumped emitters have been realized, and no DEQD-based optoelectronic device has been demonstrated up to now.

In this contribution, we report on the incorporation of Ge-DEQDs into Si $p-i-n$ junctions and demonstrate lightemitting diodes (LEDs) operating at near-infrared (NIR) wavelengths in the 1.3-1.5 $\mu \mathrm{m}$ telecommunication range. EL devices were fabricated from different active materials with a varying number of DEQD layers. The realized LEDs exhibit robust optoelectronic characteristics up to high temperatures and high current densities, and the device performance scales well with the number of stacked quantum dot layers. The presented experimental results confirm the immense potential of Ge-DEQDs as a gain material for electrically pumped group-IV lasers and pose 

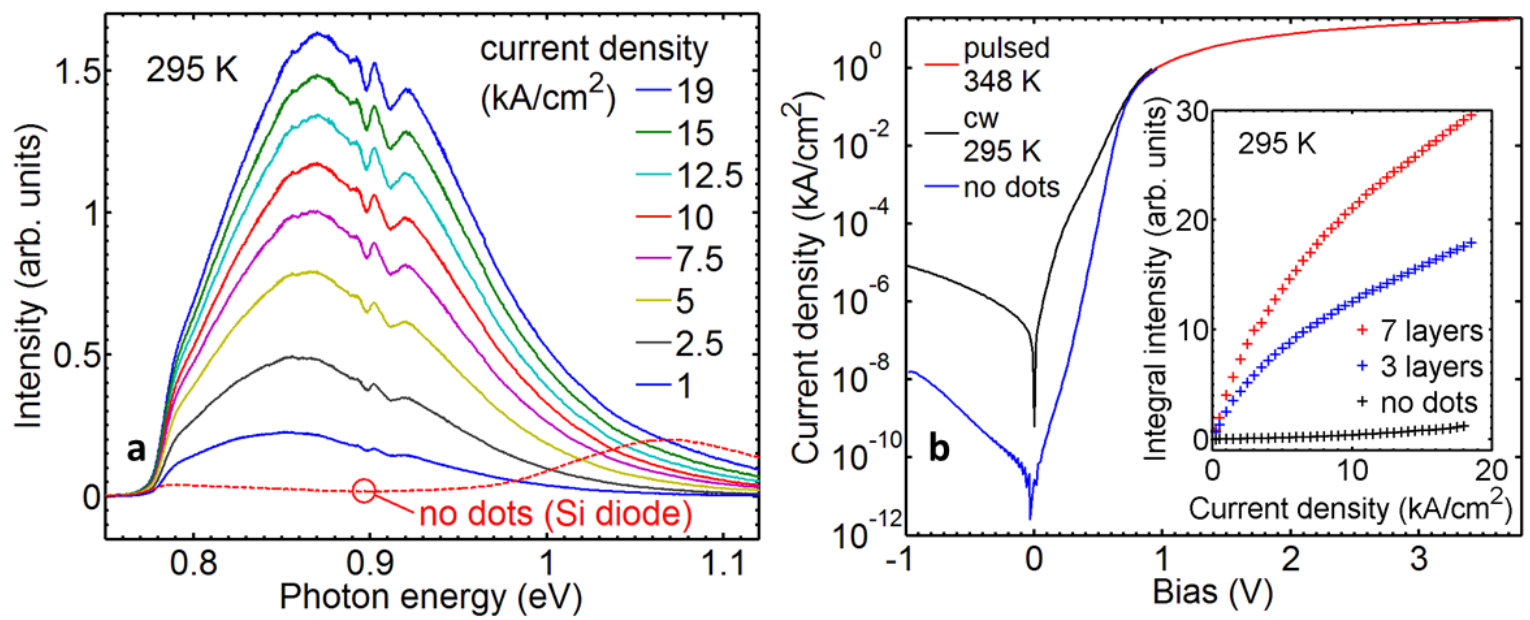

Figure 2. Room-temperature characteristics of Ge-DEQD LEDs. (a) Current-dependent EL spectra for the LED with seven layers of embedded quantum dots at a heat-sink temperature of $22^{\circ} \mathrm{C}(295 \mathrm{~K})$. The spectral peak position and width is typical of the Ge-DEQD system. The broken red line presents the emission spectrum of the Si reference diode without quantum dots at a driving current density of $10 \mathrm{kA} / \mathrm{cm}^{2}$. The spectral dips around $0.9 \mathrm{eV}$ are identified as water absorption lines. (b) Electronic characteristics of the 7-layer LED in comparison to those of the Si reference. The black and blue curves show the low-current behavior of the DEQD LED and the reference, respectively, measured under cw operation. The red curve presents the high-current characteristics of the 7 -layer LED under pulsed operation and at a temperature of $75^{\circ} \mathrm{C}(348 \mathrm{~K})$ to compensate for the less pronounced device heating in comparison to the cw characteristics. The inset compares the roomtemperature light-current characteristics of the 7- and 3-layer LEDs, demonstrating the good scaling of the integral emission intensity with the number of incorporated dot layers. In black, the integral emission curve is presented for the reference diode, once more confirming the origin of the strong DEQD LED emission from quantum dot transitions.

an essential step towards the realization of a CMOScompatible, monolithic optoelectronic platform for roomtemperature operation.

\section{-EXPERIMENTAL RESULTS}

Active Material and Device Fabrication. The studied optically active group-IV material is based on GeDEQDs, for which optically pumped lasing has been recently demonstrated ${ }^{15}$. For electrical pumping of GeDEQDs, the well-established concept of dot-incorporation into diode structures was employed. While lasing has been demonstrated on a microdisk resonator with an embedded single layer of epitaxial DEQDs, an increase in the active material volume by stacking of several dot layers has very recently been achieved ${ }^{40}$. The EL studies presented here were therefore performed on material including vertically stacked layers of dots, which were incorporated into the $200 \mathrm{~nm}$ thick intrinsic region of a $p-i-n \mathrm{Si}$ diode. The nominal doping concentrations of the $p$ and $n$ regions are

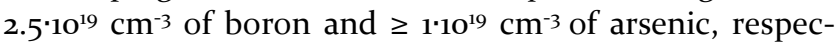
tively. Details on the material growth are given in the METHODS section. In order to confirm the scalability of the active material volume for electrically driven devices, DEQD material with 3- and 7-layer stacks was grown along with a reference diode not containing quantum dots. The fabricated material was processed into mesa devices for vertical current driving as detailed in the METHODS section. The devices feature a surface area of $100 \times 100 \mu \mathrm{m}^{2}$, limiting the current densities accessible by our driving source to $20 \mathrm{kA} / \mathrm{cm}^{2}$ at a current of $2 \mathrm{~A}$. The $p$-type top region of the $p-i-n$ diodes was contacted by an Al metallization in frame geometry to enable surface normal emission of the LEDs. A schematic representation of the fabricated devices is shown in Figure $1 \mathrm{~B}$.

Optoelectronic Characterization. The performance of the Ge-DEQD LEDs was studied under pulsed driving conditions at a repetition frequency of $10 \mathrm{kHz}$ and a current pulse length of $5 \mu \mathrm{s}$. It has to be mentioned that the duty cycle of $5 \%$ was limited by the temperature stability of the applied bonding wires at the maximum driving current of $2 \mathrm{~A}$. Further note that the current density values given throughout this publication refer to those present during the driving pulses (the average current flow is by a factor of 20 lower). In order to allow a reliable and efficient temperature-dependent device characterization both above room temperature and at cryogenic temperatures, two different setup configurations were used: (i) The LED chip was mounted on a copper block temperature-controlled by a Peltier element to access heat-sink temperatures between 280 and $380 \mathrm{~K}$. (ii) The chip carrier 


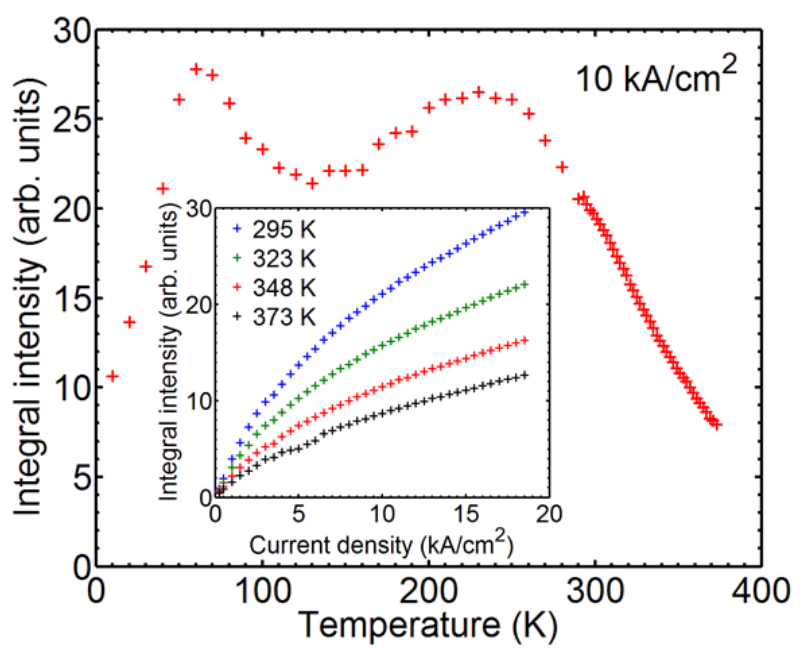

Figure 3. Temperature-dependent performance of the 7layer DEQD LED. The integral intensity at a driving current density of $10 \mathrm{kA} / \mathrm{cm}^{2}$ is shown as a function of the heat-sink temperature. Emission quenching sets in above $230 \mathrm{~K}$, but the LED performs well up to at least $100^{\circ} \mathrm{C}(373$ $\mathrm{K})$. Note the strong increase in emission intensity between 10 and $60 \mathrm{~K}$, which is related to an increase in filling efficiency of DEQD states. The inset shows light-current characteristics for a series of high temperatures, where only weak intensity saturation with increasing current is observed over the studied temperature range.

was placed in a helium-cooled cryostat for reaching temperatures between $10 \mathrm{~K}$ and $320 \mathrm{~K}$. Independent of the mounting configuration, the surface emission from the electrically driven LEDs was collimated and focused onto the entrance slit of a grating spectrometer equipped with an InGaAs line detector.

Room-Temperature Electroluminescence. The room-temperature performance of DEQD LEDs was studied in Peltier-cooled configuration at a heat-sink temperature of $22^{\circ} \mathrm{C}(295 \mathrm{~K})$. Figure $2 \mathrm{~A}$ presents the emission spectra of the 7-layer LED at a series of driving current densities. The observed NIR emission at energies below the $\mathrm{Si}$ bandgap peaks around $870 \mathrm{meV}$ consistent with the PL and lasing spectra reported for DEQDs ${ }^{15,39,4^{\circ}}$, and therefore unambiguously originates from optical transitions between quantum dot states. The identification of the signal as DEQD emission is further confirmed by the spectrum obtained for the dot-free reference diode at a driving current density of $10 \mathrm{kA} / \mathrm{cm}^{2}$, which is presented by the broken line in Figure 2A. This reference spectrum does not show any peak in the quantum dot region, but features a clear emission signal from interband transitions across the $\mathrm{Si}$ bandgap as well as a weak onset of low-energetic blackbody radiation. At room temperature, the DEQD EL peaks are relatively broad with a full-width at half-maximum (FWHM) increasing slightly from 150 to $160 \mathrm{meV}$ with the driving current. Broad-band emission is common for DEQDs and Ge dots in general due to the size distribution of the dot ensemble and the occupation of excited hole levels at elevated temperatures around $300 \mathrm{~K}$. The EL maximum in Figure $2 \mathrm{~A}$ shifts slightly with increasing driving current towards higher energies, a finding which is consistent with the pump-power-dependent peak wavelength observed in PL experiments ${ }^{15}$. The shift of the spectral peak position is less pronounced for the EL data presented here than for the reported PL results ${ }^{15}$, which is probably due to the counteracting effects of stronger device heating in case of electrical pumping.

A key observation from the EL characteristics on our GeDEQD LEDs is the continuous increase of the emission intensity with the driving current up to high densities of 19 $\mathrm{kA} / \mathrm{cm}^{2}$. This finding is highlighted by the light-current (LI) characteristics in the inset of Figure $2 \mathrm{~B}$, which shows the integral emission intensity as a function of the driving current density. The integral intensity values were obtained by using the spectrometer grating as a mirror and recording the response signal of the central InGaAs detector element. Although the rise of the LI characteristics of the DEQD LEDs is sublinear due to a current-induced increase in the device temperature, no strong saturation effects are observed. Therefore, the feasibility of driving at far higher current densities than those accessed here is anticipated, which is essential for the future realization of an electrically pumped DEQD laser. In addition to the LI curve for the 7 -layer diode, the inset of Figure $2 \mathrm{~B}$ presents the characteristics of the 3-layer material as well as the reference $\mathrm{Si}$ diode. The latter once again shows only weak emission from interband transitions in bulk Si. A comparison between the data of the 3- and 7-layer LEDs indicates that an increase in the number of dot layers by a factor of 2.3 leads to an emission intensity gain by a factor of 1.65 without any change in the LI curve shape. This experimental demonstration of emission power scaling by dot layer stacking in current-driven devices is essential for the future development of electrically pumped DEQD laser sources. Under ideal pumping conditions, the emission intensity is expected to scale linearly with the number of dot layers. However, under the given experimental conditions the individual emitter layers compete for charge carriers and the dependence is sub-linear, an effect which can be mitigated by stronger pumping and an optimization of the diode structure in future emitter systems.

Complementary to the emission performance of DEQD LEDs their current-voltage (IV) characteristics were studied in order to exclude potential fabrication-related current leakage channels, which would degrade the dot pumping efficiency. Figure $2 \mathrm{~B}$ compares the low-current $(<1$ $\mathrm{kA} / \mathrm{cm}^{2}$ ) IV curve of a 7-layer DEQD device with that of the $\mathrm{Si}$ reference diode. The incorporation of dots raises the reverse current of the fabricated diode by nearly three orders of magnitude, most likely due to an increase in edge and generation currents. However, under forward biasing and in the regime relevant for LED driving $\left(>100 \mathrm{~A} / \mathrm{cm}^{2}\right)$, the diode characteristics are only insignificantly influenced by the presence of quantum dots. Note that the low-current characteristics were obtained by a source-measure-unit 
under continuous wave (cw) operation, as the employed pulsed driver does not allow precise sourcing in this current regime. Figure $2 \mathrm{~B}$ further shows the high-current characteristics of the 7-layer DEQD LED obtained under pulsed operation. To compensate for the difference in device heating between cw and pulsed mode for an approximation of the complete IV curve between -1 and $+4 \mathrm{~V}$, the pulsed characteristics are shown for a heat-
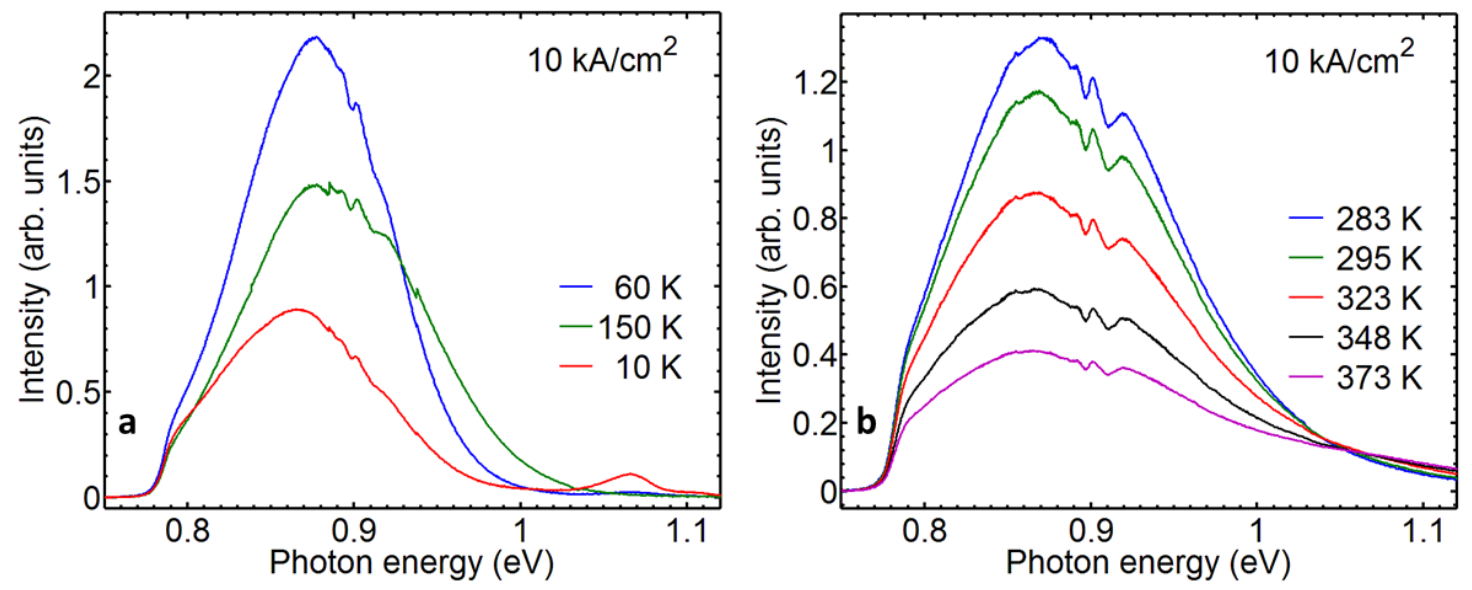

Figure 4. Temperature-dependence of the DEQD EL spectra at a driving current density of $10 \mathrm{kA} / \mathrm{cm}^{2}$. (a) The spectrally resolved EL of the 7-layer LED is presented for a series of low temperatures controlled in a helium cryostat. At $10 \mathrm{~K}$, a pronounced emission originating from bulk Si transitions is observed which is due to inefficient filling of the DEQD states. A temperature raise to $60 \mathrm{~K}$ results in a drastic increase in dot $\mathrm{EL}$ along with a quenching of the Si-related emission. A further rise in temperature leads to a broadening of the DEQD emission. (b) Spectral evolution of the 7-layer LED emission around and above room temperature in Peltier-controlled operation. A slow quenching of the DEQD EL is accompanied by a moderate spectral broadening. Note the robust LED operation at temperatures as high as $100^{\circ} \mathrm{C}(373 \mathrm{~K})$ and high current densities of $10 \mathrm{kA} / \mathrm{cm}^{2}$.

sink temperature of $75^{\circ} \mathrm{C}(348 \mathrm{~K})$. Note that the match between the black and red curves in Figure $2 \mathrm{~B}$ indicates a device temperature of at least $75^{\circ} \mathrm{C}$ for cw driving at $100 \mathrm{~mA}$. The room-temperature EL results on the first generation of DEQD LEDs already substantiate the vast potential of this group-IV system for the future demonstration of a practical laser source. To further highlight the superior temperature stability of the material performance, the temperature dependence of the LED characteristics was experimentally evaluated both at cryogenic temperatures and above $300 \mathrm{~K}$.

Temperature-Dependent Performance. The dependence of the integral emission intensity on the heatsink temperature is presented in Figure 3 for the 7 -layer LED. As seen in the plot, the intensity remains above $75 \%$ of the observed global maximum between 60 and $230 \mathrm{~K}$, before temperature quenching of the emission due to a depopulation of the confined hole states (see Figure $1 \mathrm{~A}$ ) sets in. This behavior is consistent with the DEQD PL characteristics reported in reference $\mathbf{1 5}$. For the LEDs presented here we observe an onset of emission quenching at lower heat-sink temperatures than for the optically pumped emitters reported up to now ${ }^{15}$ : While for the maximum optical pumping power of $1600 \mu \mathrm{W}$ employed in reference 15 quenching set in at $300 \mathrm{~K}$, the EL intensity drops significantly above $240 \mathrm{~K}$ at a driving current of $1 \mathrm{~A}$. This is related to the far higher actual device temperature in case of current driving. The discrepancy between heat-sink and device temperature is dependent on both the driving current value and on the heat-sink temperature. However, even for a large driving current density of $10 \mathrm{kA} / \mathrm{cm}^{2}$, significant temperature quenching of the DEQD LED emission only sets in above $240 \mathrm{~K}$, which is extremely late in comparison to the reported direct-bandgap GeSn or SiGeSn materials $^{14,21}$. Furthermore, the output intensity of the DEQD LEDs drops slowly with temperature and still remains at $28 \%$ of the global maximum value for an impressive heatsink temperature of $100^{\circ} \mathrm{C}(373 \mathrm{~K})$. The remarkable temperature stability of Ge-DEQD emission is based on the strong confinement of holes in the quantum dot potential (see Figure $1 \mathrm{~A}$ ), with associated activation energies for thermal quenching above $300 \mathrm{meV}^{39}$. The extraction of accurate activation energy values from the data in Figure 3 is prevented by the inaccessibility of the actual device temperature as opposed to the given heat-sink temperature. This is discussed in more detail as Supporting information. Note that the high-temperature characteristics in Figure 3 are dominated by the depopulation of hole states in the quantum dots, while electrons occupying the localized split[10o] interstitial levels are energetically far weaker confined.

At cryogenic conditions, the temperature dependence of the emission intensity shows a more complex behavior most likely due to the filling and emptying of the DEQD states. The significant rise of the emission intensity between 10 and $60 \mathrm{~K}$ seen in Figure 3 is either related to thermally assisted tunneling through strain-field induced 
valence band barriers flanking the dot potential well ${ }^{41}$, or is due to the more efficient electron injection into split-[10o] interstitial states at high carrier temperatures. While the shallow local minimum in the temperature-dependent emission characteristics around $140 \mathrm{~K}$ was also observed in PL experiments on DEQDs ${ }^{15}$, the significant initial increase of the intensity by more than a factor of two has only been observed in LEDs. The reason for this can be found in the short pumping wavelength used in PL experiments so far, which in contrast to electrical pumping generates charge carriers high up in the conduction or valence band.

The occupation-related lower DEQD emission at $10 \mathrm{~K}$ is accompanied by a relatively strong contribution from interband transitions in the bulk Si matrix to the PL spectrum shown in Figure 4A. As the heat-sink temperature is raised from $10 \mathrm{~K}$ to $60 \mathrm{~K}$, the DEQD emission drastically increases while the $\mathrm{Si}$ bulk contribution above $1.05 \mathrm{eV}$ quenches, clearly indicating the efficient carrier injection into DEQD states at the expense of carriers available in the surrounding $\mathrm{Si}$ matrix. At a heat-sink temperature of $60 \mathrm{~K}$ and a driving current density of $10 \mathrm{kA} / \mathrm{cm}^{2}$, the inhomogeneously broadened DEQD spectrum exhibits a FWHM of $100 \mathrm{meV}$. When further increasing the device temperature, higher-energetic DEQD states are occupied and the emission spectrum broadens towards larger photon energies in combination with a drop in spectral peak intensity, as seen in Figure 4A. The EL spectra recorded for the 7-layer DEQD LED at heat-sink temperatures around and above room temperature show further but moderate broadening of the dot emission with temperature (see Figure $4 \mathrm{~B}$ ). However, no drastic change in the spectral characteristics during emission quenching was observed up to the maximum accessible temperature of $100^{\circ} \mathrm{C}$. This is another crucial observation supporting the strong suitability of $\mathrm{Ge}$ DEQDs as a laser gain material.

The demonstration of robust DEQD LED performance at high temperatures is finally completed by the temperaturedependent LI characteristics presented in the inset of Figure 3. The curves obtained for a series of temperatures show no significant change in shape and only weak emission saturation with increasing current independent of the heat-sink temperature. Ge-DEQDs therefore maintain their optical properties under electrical pumping up to high current densities of at least $20 \mathrm{kA} / \mathrm{cm}^{2}$ and high heatsink temperatures up to $100^{\circ} \mathrm{C}$.

\section{-DISCUSSION AND OUTLOOK}

The comprehensive data set on the emitter performance of Ge-DEQD LEDs presented in this publication allows a series of important conclusions regarding the suitability of this material system for future electrically pumped groupIV lasers.

The most essential observation is the outstanding temperature stability of the optical properties of DEQDs even under electrical pumping. The temperature robustness already demonstrated for optically pumped emitters ${ }^{15}$ leads to an excellent LED performance in spite of the strong ohmic heating commonly plaguing current-driven devices. The DEQD LEDs demonstrated here operate well at current densities up to $20 \mathrm{kA} / \mathrm{cm}^{2}$, heat-sink temperatures up to $100^{\circ} \mathrm{C}$, and duty-cycles up to $5 \%$. It has to be pointed out once more that the maximum values for each of the listed parameters were limited by the employed experimental setup, either by the pulsed current source, the Peltier temperature controller or by the strength and number of bonding wires that could be conveniently attached to the devices. It can be expected with high confidence that the optically active material can operate at far higher current densities and duty cycles than those explored here. The temperature stability of the emission performance is particularly crucial for electrical pumping of laser resonators relying on a buried oxide layer for optical confinement of the laser modes to the gain material. In such a configuration, the oxide layer will pose an unwanted bottleneck for heat transfer between the emitter devices and the heatsink, calling for a temperature-stable gain material. Note that the buried oxide layer will also prevent current driving via the substrate, requiring lateral current feeding of future laser devices. The latter is again associated with ohmic heating, further increasing the importance of temperature stability.

Furthermore, for achieving a large modal overlap between guided modes and gain material in future laser devices, the volume of the active DEQD material can be conveniently increased by a stacking of quantum dot layers. As demonstrated here, the emission performance of LED devices scales with the number of incorporated dot layers, and the gain material volume is only limited by growth time considerations. Another path towards further improved material performance is the optimization of the diode structure employed for pumping of the Ge quantum dots. For the first generation of DEQD LEDs presented here no particular effort was invested in evaluating the doping parameters and intrinsic region thickness ideal for an optimized optical recombination current in the DEQDs. Nevertheless, a comparison of the characteristics of the DEQD LEDs with PL data on actual DEQD laser material ${ }^{15}$ suggests that electrically pumped DEQD lasing is within grasp even for the non-optimized first-generation diodes presented here: For the same collection efficiency, under maximum accessible pumping conditions and at room temperature, the material for which lasing in a microdisk resonator has been demonstrated ${ }^{15}$ and the 7 -layer LED presented here deliver roughly the same time-averaged emission. Considering the difference in duty-cycle (cw for optical pumping, $5 \%$ for the LED), the DEQD LED achieves a 20 times higher emission intensity during a current driving pulse with $19 \mathrm{kA} / \mathrm{cm}^{2}$ than the PL sample under optical excitation sufficient for lasing in a microdisk cavity. (Details on the comparison are given as Supporting Information.) Therefore, even with the reduced thermal conductance in buried-oxide-based cavities, the electrically driven DEQD material presented here is expected to be capable of lasing in microdisk or ridge resonators. 
To conclude, the performance of the realized Ge-DEQD LEDs demonstrates that efficient current driving of DEQD emitters is feasible and that the excellent optical properties of these unique group-IV dots are maintained under electrical pumping. The studied active material system is CMOS-compatible and the implementation of chemical vapor deposition growth of Ge quantum dots and subsequent ion bombardment in standard CMOS production lines can be envisioned 37 . The demonstrated currentdriven dot emitters are therefore highly suitable for the future realization of practical, electrically pumped group-IV lasers at room temperature. Such laser sources will form the basis for a monolithic optoelectronic platform enabling superior data transfer rates and novel optical sensing functionalities for a vast number of applications.

\section{-METHODS}

Material Growth. The studied DEQD material was grown by molecular beam epitaxy (MBE) on $n$-type substrates (highly As-doped $>1 \cdot 10^{19} \mathrm{~cm}^{-3}$ ) in a Riber SIVA45 solid-source system. The doped substrates provide the $n$ region of the fabricated $p-i-n$ diodes. After ex-situ sample cleaning and removal of the native $\mathrm{SiO}_{2}$ during a dip in diluted hydrofluoric acid, the substrates were in-situ degassed for $25 \mathrm{~min}$ at $720^{\circ} \mathrm{C}$. On the cleaned substrates, the $200 \mathrm{~nm}$ thick intrinsic region of the LED diodes was grown at $500^{\circ} \mathrm{C}$. A varying number of quantum dot layers was embedded in the center of this intrinsic region, where the individual DEQD layers were separated by $24 \mathrm{~nm}$ thick $\mathrm{Si}$ spacers. For each Ge-DEQD layer, 7.5 Å of Ge were deposited at $500^{\circ} \mathrm{C}$, and the material was bombarded with positively charged Ge ions accelerated by a bias of $-1.5 \mathrm{kV}^{15,39}$. During spacer layer growth, the substrate temperature was ramped up within the first $4 \mathrm{~nm}$ from $475^{\circ} \mathrm{C}$ to $500^{\circ} \mathrm{C}$ to achieve optimal DEQD overgrowth ${ }^{39,40}$. This procedure ensures a temperature high enough for crystalline regrowth and split-[110] self-interstitial formation 39 of the Ge quantum dot regions that were initially amorphized by ion bombardment. On the other hand, the chosen temperatures are low enough so that the resulting defect cannot migrate out of the quantum dot 39 . In the course of this work, DEQD LED material containing three and seven layers of quantum dots was grown along with a reference diode of the same geometry fabricated without the DEQD layers. The $p$-type region of the diodes was formed by 200 $\mathrm{nm}$ of boron-doped $\left(2.5 \cdot 10^{19} \mathrm{~cm}^{-3}\right) \mathrm{Si}$ deposited in a final growth step. Note that for this doping concentration, free carrier absorption of NIR emission during a single pass of the $p$-type layer is insignificant. However, future laser ridge devices will require a thorough optimization of both the applied material doping and the waveguide losses introduced by metallization layers.

Device Processing. The MBE-grown active material was processed into square-shaped mesa devices for vertical current driving using standard Si processing technology and optical lithography for structural patterning. In a first step, a $200 \mathrm{~nm}$ thick Al top metallization was deposited and structured via lift-off. The device mesas were defined by dry-etching in $\mathrm{SF}_{6}$ and $\mathrm{O}_{2}$ masked by photoresist. Another $200 \mathrm{~nm}$ thick $\mathrm{Al}$ metallization was applied as bottom contact to the substrate backside. The device chips were then mounted epilayer-up onto chip carriers by indium bonding. Aluminum wire-bonding completed the device fabrication sequence. Note that due to the fact that no sidewall isolation was applied to the LED mesas, wire bonding directly to the device top surface was required. The resulting limited space for contacting allowed the application of at most two bonding wires for each device, in turn restricting the average current values accessible. Therefore, for a peak current of $2 \mathrm{~A}$, a maximum duty cycle of $5 \%$ of the driving pulses was feasible. At higher average currents, the wire bond connections melted. For future devices with sidewall isolation and the possibility to implement bonding pad areas exceeding the device surface area, driving at higher average currents and duty cycles will be possible.

Electroluminescence Characterization. The DEQD LEDs were driven by a pulsed voltage source at a frequency of $10 \mathrm{kHz}$ and a duty cycle of $5 \%$. For this purpose, a voltage pulse was applied to the mesa top contact in respect to the grounded bottom metallization. The actual voltage drop across the device was monitored along with the driving current, which was measured using an inductive current probe. The device temperature was controlled either by a Peltier element or in a liquid-heliumcryostat, as discussed in the main text. The surface-normal emission of the LEDs was collected by a $\mathrm{CaF}_{2}$ lens with a numerical aperture of $\mathrm{NA}=2$, and the resulting parallel beam was focused onto the entrance slit of a grating spectrometer by a second lens. As the broad quantum dot emission does not require a high spectral resolution, the spectrometer slit was fully opened and the resolution limited by the size of the device image. The collected device emission was dispersed by a grating and recorded by a liquidnitrogen-cooled InGaAs line detector with a responsivity cut-off around $790 \mathrm{meV}$. The latter is visible in the spectral plots throughout this publication, but lies far below the emission peak energy and therefore only cuts off a small section of the EL spectra. As the spectral peak shifts only very weakly with temperature and driving current, the influence of the detector cut-off on the integral intensity characteristics in Figures $2 \mathrm{~B}$ and 3 is insignificant. Note that the optical path was exposed to atmosphere, resulting in the presence of water vapor absorption lines around 900 $\mathrm{meV}$ in the obtained spectra. The intensity values throughout this publication are given in 1000 counts per second as measured by the individual line detector elements. Note that a quantitative comparison between the observed DEQD-EL intensities and the EL and PL performance reported for various quantum dot systems in literature is infeasible due to the common unavailability of calibrated high-sensitivity spectroscopy systems. The latter is also required for evaluating the external quantum efficiency of the exploited radiative transitions, which therefore cannot 
be provided at the current stage. However, a detailed comparison between the EL and PL performance of the GeDEQD system is given as Supporting Information.

\section{ASSOCIATED CONTENT}

Supporting Information. Comparison between photo- and electroluminescence of Ge-DEQDs. This material is available free of charge via the Internet at http://pubs.acs.org.

\section{AUTHOR INFORMATION}

\section{Corresponding Author}

*E-mail: moritz.brehm@jku.at.

\section{ACKNOWLEDGMENT}

The authors gratefully acknowledge A. Rastelli for support. The authors acknowledge support from the Linz Institute of Technology (LIT): LIT-2016-1- YOU-oo2, and the Austrian Science Fund (FWF): P29137-N36, and P28409- N36.

\section{ABBREVIATIONS}

DEQD, defect-enhanced quantum dot; EL, electroluminescence; LED, light-emitting diode; NIR, near-infrared; FWHM, full-width at half-maximum; LI, light-current; IV, currentvoltage; cw, continuous wave; MBE, molecular beam epitaxy.

\section{REFERENCES}

(1) Rickman, A. The Commercialization of Silicon Photonics. Nat. Photonics 2014, 8, 579-582.

(2) Baldassarre, L.; Sakat, E.; Frigerio, J.; Samarelli, A.; Gallacher K.; Calandrini, E.; Isella, G.; Paul, D. J.; Ortolani, M.; Biagioni, P. Midinfrared Plasmon-Enhanced Spectroscopy with Germanium Antennas on Silicon Substrates. Nano Lett. 2015, 15, 7225-7231.

(3) Juan-Colás, J.; Parkin, A.; Dunn, K. E.; Scullion, M. G.; Krauss, T. F.; Johnson, S. D. The Electrophotonic Silicon Biosensor. Nat. Commun. 2016, 7, 12769.

(4) Chaisakul, P.; Marris-Morini, D.; Frigerio, J.; Chrastina, D.; Rouifed, M.-S.; Cecchi, S.; Crozat, P.; Isella, G.; Vivien, L. Integrated Germanium Optical Interconnects on Silicon Substrates. Nat. Photonics 2014, 8, 482-488.

(5) Debnath, K.; Gardes, F. Y.; Knights, A. P.; Reed, G. T.; Krauss, T. F.; O'Faolain, L. Dielectric Waveguide Vertically Coupled to All-Silicon Photodiodes Operating at Telecommunication Wavelengths. Appl. Phys. Lett. 2013, 102, 171106.

(6) Stern, B.; Zhu, X.; Chen, C. P.; Tzuang, L. D.; Cardenas, J.; Bergman, K.; Lipson, M. On-Chip Mode-Division Multiplexing Switch. Optica 2015, 2, 530-535.

(7) Rouifed, M.-S.; Marris-Morini, D.; Chaisakul, P.; Frigerio, J.; Isella, G.; Chrastina, D.; Edmond, S.; Le Roux, X.; Coudevylle, J.-R.; Bouville, D.; Vivien, L. Advances Toward Ge/SiGe

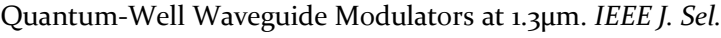
Top. Quantum Electron. 2014, 20, 33-39.

(8) Moss, D. J.; Morandotti, R.; Gaeta, A. L.; Lipson, M. New CMOS-Compatible Platforms Based on Silicon Nitride and Hydex for Nonlinear Optics. Nat. Photonics 2013, 7, 597-607.

(9) Michel, J.; Liu, J.; Kimerling, L. C. High-Performance Ge-onSi Photodetectors. Nat. Photonics 2010, 4, 527-534.

(10) Dumas, D. C. S.; Gallacher, K.; Rhead, S.; Myronov, M.; Leadley, D. R.; Paul, D. J. Ge/SiGe Quantum Confined Stark Effect Electro-Absorption Modulation with Low Voltage Swing at $\Lambda=1550 \mathrm{om}$. Opt. Express 2014, 22, 19284-19292.

(11) Takahashi, Y.; Inui, Y.; Chihara, M.; Asano, T.; Terawaki, R.; Noda, S. A Micrometre-Scale Raman Silicon Laser with a
Microwatt Threshold. Nature 2013, 498, 470-474

Süess, M. J.; Geiger, R.; Minamisawa, R. A.; Schiefler, G.; Frigerio, J.; Chrastina, D.; Isella, G.; Spolenak, R.; Faist, J.; Sigg, H. Analysis of Enhanced Light Emission from Highly Strained Germanium Microbridges. Nat. Photonics 2013, 7 , 466-472.

(13) Liu, J.; Sun, X.; Camacho-Aguilera, R.; Kimerling, L. C.; Michel, J. Ge-on-Si Laser Operating at Room Temperature. Opt. Lett. 2010, 35, 679-681.

(14) Wirths, S.; Geiger, R.; von den Driesch, N.; Mussler, G.; Stoica, T.; Mantl, S.; Ikonic, Z.; Luysberg, M.; Chiussi, S.; Hartmann, J. M.; Sigg, H.; Faist, J.; Buca, D.; Grützmacher, D. Lasing in Direct-Bandgap GeSn Alloy Grown on Si. Nat. Photonics 2015, 9, 88-92.

(15) Grydlik, M.; Hackl, F.; Groiss, H.; Glaser, M.; Halilovic, A.; Fromherz, T.; Jantsch, W.; Schäffler, F.; Brehm, M. Lasing from Glassy Ge Quantum Dots in Crystalline Si. ACS Photonics 2016, 3, 298-303.

(16) Shakoor, A.; Lo Savio, R.; Cardile, P.; Portalupi, S. L.; Gerace, D.; Welna, K.; Boninelli, S.; Franzo, G.; Priolo, F.; Krauss, T. F.; Galli, M.; O'Faolain, L. Room Temperature All-Silicon Photonic Crystal Nanocavity Light Emitting Diode at SubBandgap Wavelengths. Laser Photon. Rev. 2013, 7, 114-121.

(17) Mayer, B.; Janker, L.; Loitsch, B.; Treu, J.; Kostenbader, T.; Lichtmannecker, S.; Reichert, T.; Morkötter, S.; Kaniber, M.; Abstreiter, G.; Gies, C.; Koblmüller, G.; Finley, J. J. Monolithically Integrated High- $\beta$ Nanowire Lasers on Silicon. Nano Lett. 2016, 16, 152-156.

(18) Chen, S.; Li, W.; Wu, J.; Jiang, Q.; Tang, M.; Shutts, S.; Elliott, S. N.; Sobiesierski, A.; Seeds, A. J.; Ross, I.; Smowton, P. M.; Liu, H. Electrically Pumped Continuous-Wave III-V Quantum Dot Lasers on Silicon. Nat. Photonics 2016, 10, 307-311.

(19) Wang, Z.; Tian, B.; Pantouvaki, M.; Guo, W.; Absil, P.; Van Campenhout, J.; Merckling, C.; Van Thourhout, D. RoomTemperature InP Distributed Feedback Laser Array Directly Grown on Silicon. Nat. Photonics 2015, 9, 837-842.

(20) Homewood, K. P.; Lourenço, M. A. Optoelectronics: The Rise of the GeSn Laser. Nat. Photonics 2015, 9, 78-79.

(21) von den Driesch, N.; Stange, D.; Wirths, S.; Rainko, D.; Povstugar, I.; Savenko, A.; Breuer, U.; Geiger, R.; Sigg, H.; Ikonic, Z.; Hartmann, J. M.; Grützmacher, D.; Mantl, S.; Buca, D. SiGeSn Ternaries for Efficient Group IV Heterostructure Light Emitters. Small 2017, 13, 1-9.

(22) Stange, D.; von den Driesch, N.; Rainko, D.; Roesgaard, S.; Povstugar, I.; Hartmann, J.-M.; Stoica, T.; Ikonic, Z.; Mantl, S.; Grützmacher, D.; Buca, D. Short-Wave Infrared LEDs from GeSn / SiGeSn Multiple Quantum Wells. Optica 2017, 4, $185^{-188 .}$

(23) Stange, D.; von den Driesch, N.; Rainko, D.; Schulte-Braucks, C.; Wirths, S.; Mussler, G.; Tiedemann, A. T.; Stoica, T.; Hartmann, J. M.; Ikonic, Z.; Mantl, S.; Grützmacher, D.; Buca, D. Study of GeSn Based Heterostructures: Towards Optimized Group IV MQW LEDs. Opt. Express 2016, 24, 1358-1367.

(24) Brunhes, T.; Boucaud, P.; Sauvage, S.; Aniel, F.; Lourtioz, J.M.; Hernandez, C.; Campidelli, Y.; Kermarrec, O.; Bensahel, D.; Faini, G.; Sagnes, I. Electroluminescence of Ge/Si SelfAssembled Quantum Dots Grown by Chemical Vapor Deposition. Appl. Phys. Lett. 200o, 77, 1822-1824.

(25) de Kersauson, M.; Jakomin, R.; El Kurdi, M.; Beaudoin, G.; Zerounian, N.; Aniel, F.; Sauvage, S.; Sagnes, I.; Boucaud, P. Direct and Indirect Band Gap Room Temperature Electroluminescence of Ge Diodes. J. Appl. Phys. 2010, 108, 23105.

(26) Tsybeskov, L.; Lockwood, D. J. Silicon-Germanium Nanostructures for Light Emitters and on-Chip Optical Interconnects. Proc. IEEE 2009, 97, 1284-1303. 
(27) Tsuboi, T.; Xu, X.; Xia, J.; Usami, N.; Maruizumi, T.; Shiraki, Y. Room-Temperature Electroluminescence from $\mathrm{Ge}$ Quantum Dots Embedded in Photonic Crystal Microcavities. Appl. Phys. Express 2012, 5, 52101.

(28) Xu, X.; Maruizumi, T.; Shiraki, Y. Waveguide-Integrated Microdisk Light-Emitting Diode and Photodetector Based on Ge Quantum Dots. Opt. Express 2014, 22, 3902-3910.

(29) Lobanov, D. N.; Novikov, A. V.; Kudryavtsev, K. E.; Shaleev, M. V.; Shengurov, D. V.; Krasilnik, Z. F.; Zakharov, N. D.; Werner, P. Effect of Silicon Spacer Thickness on the Electroluminescence of Multilayer Structures with SelfAssembled $\mathrm{Ge}(\mathrm{Si}) / \mathrm{Si}(\mathrm{ool})$ Islands. Semiconductors 2012, 46, 1418-1422.

(30) Thewalt, M. L. W.; Harrison, D. A.; Reinhart, C. F.; Wolk, J. A.; Lafontaine, H. Type II Band Alignment in Si1xGex/Si(ool) Quantum Wells: The Ubiquitous Type I Luminescence Results from Band Bending. Phys. Rev. Lett. 1997, 79, 269-272.

(31) Penn, C.; Schäffler, F.; Bauer, G.; Glutsch, S. Application of Numerical Exciton-Wave-Function Calculations to the Question of Band Alignment in Si/Si1-xGex Quantum Wells. Phys. Rev. B 1999, 59 (20), 13314-13321.

(32) Dashiell, M. W.; Denker, U.; Schmidt, O. G. Photoluminescence Investigation of Phononless Radiative Recombination and Thermal-Stability of Germanium Hut Clusters on silicon(oo1). Appl. Phys. Lett. 2001, 79, 2261-2263.

(33) Abstreiter, G.; Schittenhelm, P.; Engel, C.; Silveira, E.; Zrenner, A.; Meertens, D.; Jäger, W. Growth and Characterization of Self-Assembled Ge-Rich Islands on Si. Semicond. Sci. Technol. 1996, 11, 1521-1528.

(34) Sunamura, H.; Usami, N.; Shiraki, Y.; Fukatsu, S. Island Formation during Growth of $\mathrm{Ge}$ on $\mathrm{Si}(100)$ : A Study Using Photoluminescence Spectroscopy. Appl. Phys. Lett. 1995, 66, 3024-3026.
(35) Schatzl, M.; Hackl, F.; Glaser, M.; Rauter, P.; Brehm, M.; Spindlberger, L.; Simbula, A.; Galli, M.; Fromherz, T.; Schäffler, F. Enhanced Telecom Emission from Single Group-IV Quantum Dots by Precise CMOS-Compatible Positioning in Photonic Crystal Cavities. ACS Photonics 2017, 4, 665-673.

(36) Grydlik, M.; Langer, G.; Fromherz, T.; Schäffler, F.; Brehm, M. Recipes for the Fabrication of Strictly Ordered Ge Islands on Pit-Patterned Si(oo1) Substrates. Nanotechnology 2013, 24, 105601.

(37) Brehm, M.; Grydlik, M. Site-Controlled and Advanced Epitaxial Ge/Si Quantum Dots: Fabrication, Properties, and Applications. Nanotechnology 2017, 28, 392001.

(38) Blöchl, P. E.; Smargiassi, E.; Car, R.; Laks, D. B.; Andreoni, W.; Pantelides, S. T. First-Principles Calculations of SelfDiffusion Constants in Silicon. Phys. Rev. Lett. 1993, 70, $2435^{-2438 .}$

(39) Grydlik, M.; Lusk, M. T.; Hackl, F.; Polimeni, A.; Fromherz, T.; Jantsch, W.; Schäffler, F.; Brehm, M. Laser Level Scheme of Self-Interstitials in Epitaxial Ge Dots Encapsulated in Si. Nano Lett. 2016, 16, 6802-6807.

(40) Groiss, H.; Spindlberger, L.; Oberhumer, P.; Schäffler, F.; Fromherz, T.; Grydlik, M.; Brehm, M. Photoluminescence Enhancement through Vertical Stacking of DefectEngineered Ge on Si Quantum Dots. Semicond. Sci. Technol. 2017, 32, 02LTo1.

(41) Brehm, M.; Grydlik, M.; Tayagaki, T.; Langer, G.; Schäffler, F.; Schmidt, O. G. Photoluminescence Investigation of Strictly Ordered Ge Dots Grown on Pit-Patterned Si Substrates. Nanotechnology 2015, 26, 225202. 


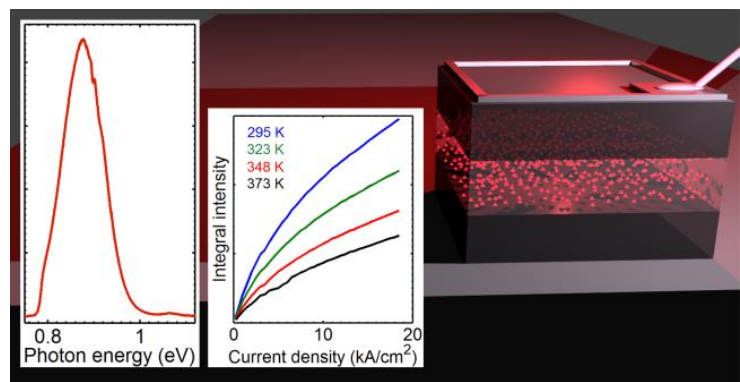

